\title{
A 20 min window is optimal in a non-wear algorithm for tri-axial thigh-worn accelerometry in overweight people
}

Citation for published version (APA):

Berendsen, B. A. J., Hendriks, M. R. C., Willems, P., Meijer, K., Schaper, N. C., \& Savelberg, H. H. C. M. (2014). A $20 \mathrm{~min}$ window is optimal in a non-wear algorithm for tri-axial thigh-worn accelerometry in overweight people. Physiological Measurement, 35(11), 2205-2212. https://doi.org/10.1088/0967$3334 / 35 / 11 / 2205$

Document status and date:

Published: 01/11/2014

DOI:

10.1088/0967-3334/35/11/2205

Document Version:

Publisher's PDF, also known as Version of record

Document license:

Taverne

Please check the document version of this publication:

- A submitted manuscript is the version of the article upon submission and before peer-review. There can be important differences between the submitted version and the official published version of record.

People interested in the research are advised to contact the author for the final version of the publication, or visit the DOI to the publisher's website.

- The final author version and the galley proof are versions of the publication after peer review.

- The final published version features the final layout of the paper including the volume, issue and page numbers.

Link to publication

\footnotetext{
General rights rights.

- You may freely distribute the URL identifying the publication in the public portal. please follow below link for the End User Agreement:

www.umlib.nl/taverne-license

Take down policy

If you believe that this document breaches copyright please contact us at:

repository@maastrichtuniversity.nl

providing details and we will investigate your claim.
}

Copyright and moral rights for the publications made accessible in the public portal are retained by the authors and/or other copyright owners and it is a condition of accessing publications that users recognise and abide by the legal requirements associated with these

- Users may download and print one copy of any publication from the public portal for the purpose of private study or research.

- You may not further distribute the material or use it for any profit-making activity or commercial gain

If the publication is distributed under the terms of Article $25 \mathrm{fa}$ of the Dutch Copyright Act, indicated by the "Taverne" license above, 
PAPER

\section{A 20 min window is optimal in a non-wear algorithm for tri-axial thigh-worn accelerometry in overweight people}

To cite this article: Brenda A J Berendsen et al 2014 Physiol. Meas. 352205

View the article online for updates and enhancements.

\section{Related content}

\author{
Validity of an automated algorithm to \\ identify waking and in-bed wear time in \\ hip-worn accelerometer data collected with \\ a $24 \mathrm{~h}$ wear protocol in young adults \\ Joanne A McVeigh, Elisabeth A H Winkler, \\ Genevieve N Healy et al. \\ - Identifying adults' valid waking wear time \\ by automated estimation in activPAL data \\ collected with a $24 \mathrm{~h}$ wear protocol \\ Elisabeth A H Winkler, Danielle H \\ Bodicoat, Genevieve N Healy et al. \\ - Empirically derived cut-points for \\ sedentary behaviour: are we sitting \\ differently? \\ Alexandra M Clarke-Cornwell, Tracey M \\ Farragher, Penny A Cook et al.
}

\section{Recent citations}

- $\frac{\text { Issues Related to Measuring and }}{\text { Interpreting Objectively Measured }}$
$\frac{\text { Sedentary Behavior Data }}{\text { Xanne Janssen and Dylan P. Cliff }}$
- $\frac{\text { 3rd international conference on }}{\text { ambulatory monitoring of physical activity }}$
$\frac{\text { and movement (University of }}{\text { Massachusetts, Amherst, USA, June }}$
$\frac{17-19,2013)}{\text { Ben Stansfield et al }}$

The goal of ECS Sensors Plus, as a one-stop shop journal for sensors, is to advance the fundamental science and understanding of sensors and detection technologies for efficient monitoring and control of industrial processes and the environment, and improving quality of life and human health.

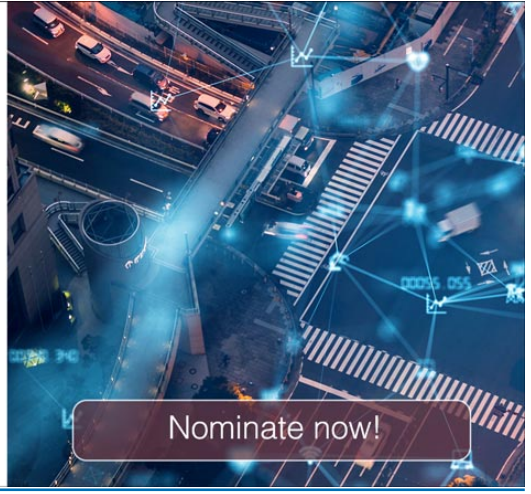




\title{
A 20 min window is optimal in a non- wear algorithm for tri-axial thigh-worn accelerometry in overweight people
}

\author{
Brenda A J Berendsen ${ }^{1}$, Marike R C Hendriks ${ }^{1,2}$, \\ Paul Willems ${ }^{1}$, Kenneth Meijer ${ }^{1}$, Nicolaas C Schaper ${ }^{3}$ and \\ Hans H C M Savelberg ${ }^{1}$ \\ 1 Human Movement Science, NUTRIM, School for Nutrition, Toxicology and \\ Metabolism, Maastricht University Medical Centre, PO Box 616, 6200 MD \\ Maastricht, The Netherlands \\ ${ }^{2}$ Public and Occupational Health, EMGO Institute for Health and Care Research, VU \\ University Medical Centre, Amsterdam, The Netherlands \\ ${ }^{3}$ Internal Medicine, CAPHRI, School for Public Health and Primary Care, Maastricht \\ University Medical Centre, Maastricht, The Netherlands
}

E-mail: Brenda.Berendsen@maastrichtuniversity.nl

Received 7 March 2014, revised 30 June 2014

Accepted for publication 21 August 2014

Published 23 October 2014

\begin{abstract}
A valid non-wear algorithm for activity monitors is crucial to avoid the misclassification of sedentary time as non-wear time, and vice versa. Characteristics of the algorithm, such as time windows, should be well defined and tested. Furthermore, using tri-axial data might influence the algorithm's performance. This study assessed the optimal time window length in a non-wear algorithm for overweight adults, applied to tri-axial data from sixteen participants. Ten time windows, from 10 up to $120 \mathrm{~min}$, were tested with a diary as a criterion measure. We assessed the bias in nonwear time, sensitivity and specificity. The optimal time window length was based on ten participants; the validation of this time window was carried out with six other participants. The time window of $20 \mathrm{~min}$ showed the highest and 120 min showed the lowest mean amount of correctly classified non-wear time, at $94 \%$ and $70 \%$ respectively. Sensitivity and specificity were considered optimal in the 20 min time window. Validation of this time window demonstrated a sensitivity and specificity of $86 \%$ and $83 \%$ respectively. A 20 min time window showed the best non-wear estimations. The current study utilized tri-axial raw data and $1 \mathrm{~s}$ epoch data which might have facilitated the application of a short time window and thereby decreased the risk of misclassifying non-wear.
\end{abstract}


Keywords: activity monitor, non-wear classification, overweight, physical activity, sedentary behaviour

(Some figures may appear in colour only in the online journal)

\section{Introduction}

Although accelerometry offers an objective measure of physical behaviour (e.g. time spent sitting or being physically active), accuracy relies on the compliance of wearing the activity monitor. For various reasons, such as water-related activities, and sleeping, participants rarely wear the activity monitor continuously. To prevent the influence of non-wear on the physical behaviour output, non-wear time should be removed from the data before analyzing the activity monitor measurements. The most commonly used algorithm classifies time as non-wear only if zero counts on the vertical axis are registered for at least 60 consecutive minutes, with allowances for 1-2 min with counts between 0 and 100 (Troiano et al 2008, Tudor-Locke et al 2010). This algorithm has also been used for NHANES data measured with a uniaxial ActiGraph worn at the waist (Troiano et al 2008). However, one non-wear algorithm cannot be applied to other activity monitors with other placements or populations without validation.

Manipulation of the time window results in different estimations of physical activity and inactivity, and therefore might affect conclusions drawn from measurements (Masse et al 2005, Healy et al 2008, Evenson and Terry 2009, Tudor-Locke et al 2010, Choi et al 2012, Miller et al 2013). The lengths of time windows differ from 10 to $90 \mathrm{~min}$ for the algorithms utilized (Evenson and Terry 2009, Choi et al 2012). In addition, studies apply a minimum wear time of $10 \mathrm{~h} \mathrm{~d}^{-1}$ to consider a measurement valid (Trost et al 2005). The utilization of an invalid algorithm can falsely decrease or increase the number of valid days due to the misclassification of non-wear. It is difficult to avoid misclassification of non-wear, especially in sedentary populations such as the elderly, because both sedentary time and non-wear time show similar outputs. In addition, Winkler et al (2012) showed that body mass index (BMI) influences the performance of the generally accepted non-wear algorithm. Non-wear misclassification was significantly higher in overweight and obese persons compared to normal and underweight persons (Winkler et al 2012), indicating that algorithms validated in normal weight persons might not be valid in overweight and obese persons. Thus, the length of the time window should be tested in the population of interest. If the study involves a population that is more likely to be sedentary, such as overweight or elderly persons, the time window should probably be longer to avoid the misclassification of sedentary time as non-wear time. Choi et al (2012) found less misclassification in the elderly when using a time window of $90 \mathrm{~min}$, compared to a time window of $60 \mathrm{~min}$. In addition, Hutto et al (2013) found longer time windows resulted in less misclassification of non-wear in older adults. However, a problem related to a larger time window is the increased risk of misclassification of short non-wear intervals (such as during showering) as wear time (Van Domelen et al 2011, Choi et al 2012). Unfortunately, conclusive evidence about the optimal time window length in overweight adults is lacking.

Until now, most non-wear algorithms have been based on activity counts on one axis (Masse et al 2005, Healy et al 2008, Evenson and Terry 2009, Choi et al 2011, Harrington et al 2011, Winkler et al 2012). Since many activity monitors measure acceleration on three axes, applying a non-wear algorithm on tri-axial data might improve non-wear estimates. In a wrist-worn activity monitor, an algorithm based on three axes performed better in estimating non-wear when compared to the algorithm based on vertical counts only (Choi et al 2012). In addition, as a wearing location the wrist was more sensitive in detecting wear and non-wear 
than the waist. The utilization of three axes in the non-wear algorithm of the waist-worn activity monitor was not assessed. Therefore it is not known whether a non-wear algorithm on triaxial data worn on locations other than the wrist might improve non-wear estimates.

Because the optimal time window is still unclear, and because including tri-axial data might improve the classification of non-wear, we aimed to find and validate the optimal time window in a non-wear algorithm in overweight and obese adults using the tri-axial CAM, worn at the thigh.

\section{Methods}

Sixteen participants of the BeweegKuur study (Berendsen et al 2011) were randomly selected. Participants were included by general practitioners, were overweight or obese, and wore the CAM (Maastricht Instruments BV, Maastricht, NL) (Annegarn et al 2011) for 5 consecutive days before participating in a combined lifestyle intervention. The CAM is a tri-axial accelerometer with a sample frequency of $25 \mathrm{~Hz}$ and a range of $4 \mathrm{G}$. The device was worn on an elastic belt around the thigh and had to be removed for sleeping and when there was a risk of getting wet. Participants were encouraged to wear the monitor continuously and to write down non-wear intervals in a diary, with reasons for non-wear and specific starting and ending times. The times in the diary were used to retrieve the exact start and end times of wear and non-wear intervals in the graphical representation of the accelerations. This method has previously been used by Choi et al (2012) to assess the accuracy of non-wear algorithms.

First, the total non-wear time was calculated based on the exact starting and ending times of the non-wear intervals, and used as a criterion measure. Second, the total measurement was divided into consecutive wear and non-wear intervals. These intervals were used to assess the sensitivity and specificity of the algorithms.

The most optimal time window was determined based on the data from ten participants (mean age $54 \pm 15$ years; mean BMI $34.0 \pm 4.6 \mathrm{~kg} \mathrm{~m}^{-2}$ ), and then validated with data from six other participants of the BeweegKuur study (mean age $63 \pm 8$ years; mean BMI $36.0 \pm 4.9 \mathrm{~kg} \mathrm{~m}^{-2}$ ).

\subsection{Data filtering and algorithm parameters}

If counts remained below the CAM's noise level for 10-120 min, time was classified as nonwear time, with an allowance of $60 \mathrm{~s}$ of counts between the noise level and the previously validated sedentary cut off point (Annegarn et al 2011 and Berendsen et al 2014). The duration of the time window was manipulated from 10 to $120 \mathrm{~min}$, in steps of $10 \mathrm{~min}$. After the classification of non-wear, the algorithm assessed the change in accelerations in the three axes between the start and end of each non-wear period. If this change reached a threshold, we assumed the accelerometer had slightly moved and the time was considered to be wear time.

\subsection{Analyses}

For both the optimization and validation of the non-wear algorithm, we compared the criterion measure with non-wear intervals estimated by the algorithm. For each time window we assessed the bias in total duration of non-wear, defined as the total duration of the criterion measure minus the total duration of non-wear estimated by the algorithm. In addition, we analyzed whether each wear and non-wear interval was correctly classified by the algorithm (with an allowed deviation of $5 \mathrm{~min}$ in the start and end time). Based on the classification per 


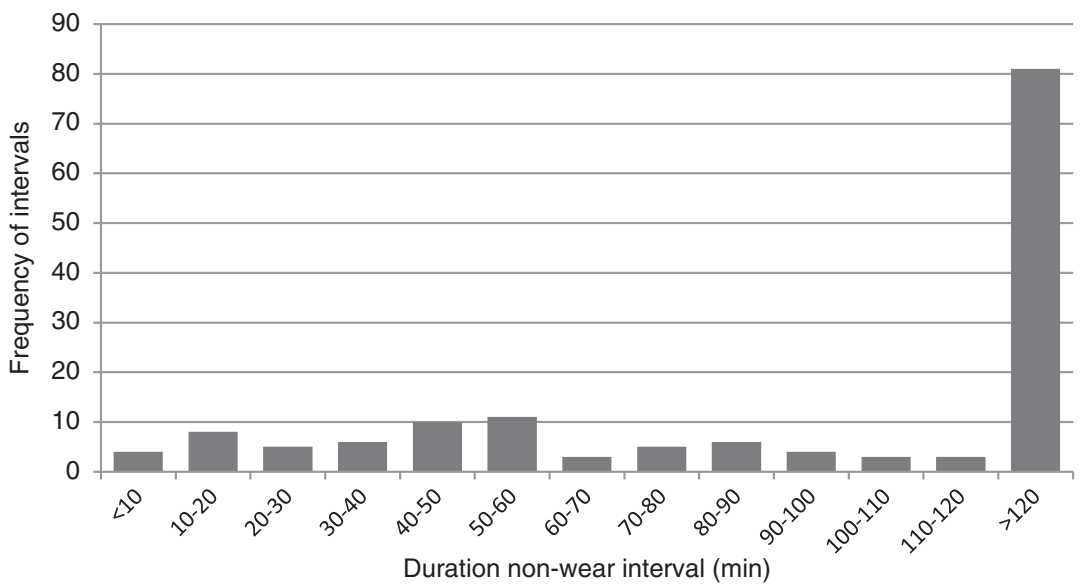

Figure 1. Frequency of non-wear intervals.

interval, sensitivity (true non-wear/all non-wear) and specificity (true wear/all wear) were calculated. A true positive was defined as a correctly classified non-wear interval; a true negative was defined as a correctly classified wear interval. The most optimal algorithm would result in the least bias between our criterion measure and the algorithm, and have an acceptable sensitivity and specificity. Analyses were performed using a custom MATLAB program.

\section{Results}

Diaries showed that the mean non-wear time due to sleeping was $34.6 \pm 9.6 \%$ of the total time measured. Non-wear due to other reasons, such as showering and skin irritation, was $3.0 \pm 3.5 \%$ of the time. Besides for sleeping, the least number of removals during the entire measurement per participant was zero; the maximal number of removals was five; and the median was two removals. Because the CAM had to be removed for sleeping, non-wear intervals longer than $2 \mathrm{~h}$ occurred most frequently. Of the non-wear intervals shorter than $2 \mathrm{~h}, 65 \%$ were under $1 \mathrm{~h}$ (figure 1$)$.

In most participants $(n=8)$, the time window of 10 or 20 min was optimal. In those participants, longer time windows led to decreases in the correctly classified non-wear time. In one participant all time windows resulted in the same amount of bias, and in one participant the $30 \mathrm{~min}$ time window was optimal for the estimation of total non-wear time. The mean percentage of non-wear that was correctly classified by the algorithm increased from $70.0 \%$ (range $0.0-99.0 \%$ ) with a time window of $120 \mathrm{~min}$, up to $94.4 \%$ (range $83.1-100.0 \%$ ) for a time window of $20 \mathrm{~min}$. The $10 \mathrm{~min}$ time window lead to $91.4 \%$ (range $82.5-99.5 \%$ ) correctly classified non-wear time (figure 2).

With increasing time window lengths, the specificity increased from 59.3 to $95.0 \%$, and the sensitivity decreased from 89.0 to $47.3 \%$. The values of $(0 ; 0)$ and $(1 ; 1)$ were added in the ROC-curve for illustration, to represent $100 \%$ sensitivity and $0 \%$ specificity and vice versa (figure 3 ). The area under the curve was 0.83 . The percentage of correctly classified non-wear time and the combination of sensitivity and specificity were deemed best for the time window of $20 \mathrm{~min}$.

The validity of the 20 min time window was assessed in six other overweight participants. The amount of non-wear time that was correctly classified by the algorithm in each 


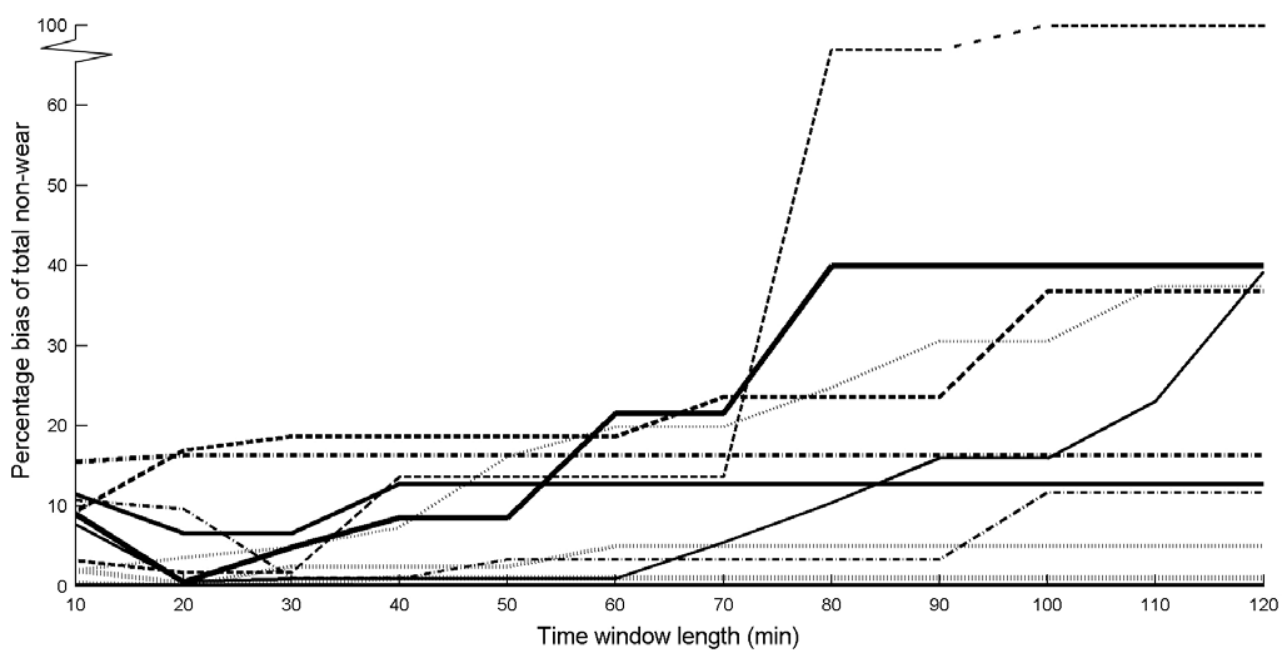

Figure 2. Percentage difference between algorithm and the criterion measure as a function of time window length, per participant (different lines).

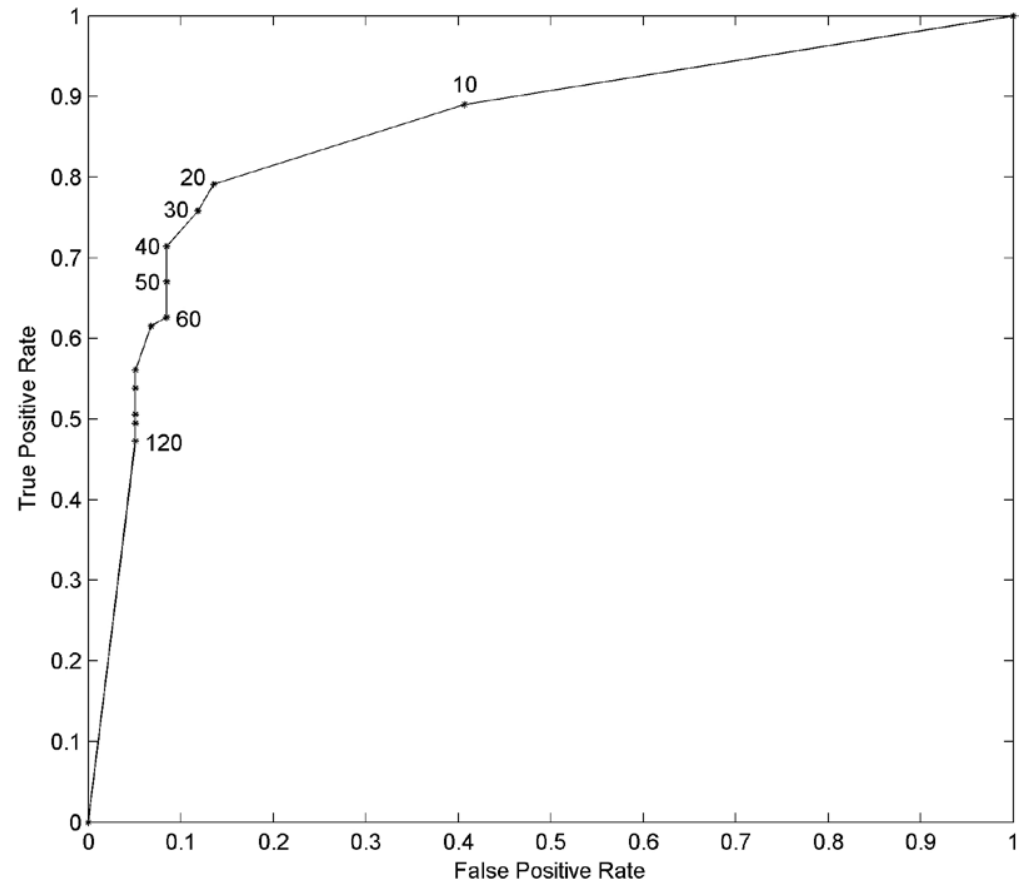

Figure 3. ROC-curve of the algorithm. The numbers next to the markers represent specific time windows.

participant ranged from 67.0 to $98.8 \%$, with a mean of $90.5 \pm 11.9 \%$. In three participants the algorithm overestimated non-wear; in the other participants the algorithm underestimated non-wear. The sensitivity and specificity of the $20 \mathrm{~min}$ algorithm were respectively $86.2 \%$ and $83.3 \%$. In one participant, the CAM provided data on only two axes which 
seemed to lead to extra false negatives and an over estimation of wear time. When we excluded the data of this participant, sensitivity increased to $92.0 \%$.

\section{Discussion}

Results of the current study showed that a time window of 20 min yielded the most accurate estimates of wear and non-wear when applying an algorithm on raw tri-axial data collected from overweight people. Commonly used algorithms have a time window of $60 \mathrm{~min}$, allow for 1-2 min with counts up to the sedentary cut off point, and utilize the counts on the vertical axis only (Troiano et al 2008, Tudor-Locke et al 2010). Recent studies have recommended time windows of 90-120 min (Choi et al 2012, Hutto et al 2013) to reduce the amount of false positives (i.e. misclassification of wear as non-wear). However, in our measurements, a substantial proportion of the non-wear intervals were shorter than an hour. This increases the risk of false negatives, i.e. non-wear misclassified as wear (Van Domelen et al 2011). Therefore, both measurement states (wear and non-wear) should be considered and analyzed when choosing and optimizing non-wear algorithms (Van Domelen et al 2011). While studies have compared the total non-wear time of the algorithm with the comparator and might have found acceptable agreement (Evenson and Terry 2009, Choi et al 2012, Hutto et al 2013), it is essential that the time excluded from analysis corresponds precisely to the actual non-wear. Therefore, validation studies should include analyses of starting and ending times of each interval to decrease the risk of excluding wear time or including non-wear time in analyses. In the current study, we calculated both the sensitivity and specificity of the algorithm to assess whether each wear and nonwear interval was classified correctly. The time window of $20 \mathrm{~min}$ led to an acceptable sensitivity and specificity in both the optimization analysis (79.1\% and $86.4 \%$ respectively) and the validation $(86.2 \%$ and $83.3 \%$ respectively). Although a larger time window showed higher specificity (i.e. less non-wear misclassified as wear) and a shorter time window showed higher sensitivity, these time windows were not chosen due to the related decreases in sensitivity or specificity. Low sensitivity would lead to incorrect analysis of data, possibly leading to overestimation of sedentary time; in contrast, low specificity would lead to wrongly excluded data, possibly leading to underestimation of sedentary time. Both situations are undesirable, and the combination of sensitivity and specificity values led to the decision to adopt a $20 \mathrm{~min}$ time window.

Previous studies often used uni-axial and pre-processed data, providing less information when compared to our tri-axial device which provided the raw data. However, one other study comparing algorithms in a tri-axial activity monitor found results similar to the studies using uni-axial data (Choi et al 2012). Moreover, the utilization of tri-axial data improved non-wear detection in the wrist-worn monitor (Choi et al 2012). We did not compare uni- with tri-axial in the current study; therefore, we cannot conclude whether the use of three axes is beneficial over one axis. As far as we know, this is the first study using raw and $1 \mathrm{~s}$ epoch data. Most activity monitors are provided with a software package, offering pre-defined filtering and calculations of counts, often summarized in minutes. Data is smoothened when using 1 min epochs, leading to a certain loss of information (Trost et al 2005). Smoothing does not only lead to altered estimations of physical activity and sedentary time, but also relates to the classification of wear and non-wear. Our raw data was summarized to $1 \mathrm{~s}$ epochs before the algorithm was applied, requiring less smoothing and providing a more accurate reflection of actual behaviour. It is probable that the small movements during sedentary time are detectable in CAM data, but not in the pre-processed data of other activity monitors. Therefore, the risk of misclassifying sedentary time as non-wear with a short time window could be decreased in our data, despite the fact that a longer time window has been hypothesized to be more valid 
in a sedentary population (Choi et al 2012, Hutto et al 2013). Although our algorithm showed lower specificity than that reported by Winkler et al (2012), it is still considered acceptable for utilization in physical behaviour analyses.

The role of placement of activity monitors in relation to the accuracy of non-wear algorithms should be further explored. The commonly used algorithm with a 60 min time window has mainly been applied to waist-worn activity monitors (Troiano et al 2008, Tudor-Locke et al 2010). Choi et al (2012) also assessed non-wear algorithms in a wrist-worn activity monitor. Although the $90 \mathrm{~min}$ time window was found to be optimal for both wearing locations (waist and wrist), the wrist location showed less bias in wear time classification (Choi et al 2012). Wrist-worn activity monitors seem to enhance compliance; however, the classification of sedentary time is challenging (Rosenberger et al 2013). The CAM is thigh-worn to enable discrimination of sedentary time from standing and active time (Annegarn et al 2011 and Berendsen et al 2014). One could argue that an activity monitor placed on an extremity (arm or leg) would be more sensitive to small movements during sedentary time, decreasing the risk of incorrectly classified non-wear (Pat Rapp et al 2010, Zhang et al 2012). However, in contrast to our findings in the thigh-worn monitor, the study concerning non-wear of the wrist-worn monitor indicated that a 90 min time window would be optimal (Choi et al 2012). A possible explanation for this discrepancy is the low amount of non-wear time in the study of Choi et al (2012). In addition, the fact that the wrist-worn device was water-proof might have decreased the probability of short non-wear periods. Unfortunately, no information was available about the duration of non-wear periods in that particular study. Therefore, although current findings indicate possibilities for the utilization of tri-axial accelerations for the identification of non-wear, research in activity monitors with other placement is required.

One strength of our study is the validation of the optimized non-wear algorithm. Optimization of an algorithm is the first step towards automated non-wear detection; validation of this algorithm in other participants is the second and often overlooked step. Only after validation can researchers make solid conclusions about algorithm accuracy. In addition, we defined our algorithm specifically for our target population: overweight adults. As the accuracy of non-wear algorithms may differ due to participant characteristics (Winkler et al 2012), it is essential to validate the algorithm in the population it will be used for.

One limitation of our study is that the results apply to the CAM specifically; however, raw and $1 \mathrm{~s}$ epoch data can be collected with other devices as well, and conclusions about the short time window might be transferrable. Although we used data from our target population, data from only sixteen participants was used. In addition, we were limited to using diaries as the criterion measure for wear and non-wear time. Nevertheless, we checked all wear and non-wear intervals in the graphical representations of the raw data, to eliminate wrongly registered intervals.

\section{Conclusion}

Based on the current optimization and validity study we conclude that the algorithm with a time window of $20 \mathrm{~min}$ is acceptably sensitive and specific for participants who are overweight. The characteristics of algorithms differ between populations, devices, device placement and dataprocessing, and should therefore be tested thoroughly in the population of interest.

\section{Acknowledgments}

The study is part of a project funded by ZonMW, The Netherlands Organization for Health Research and Development (project number: 123000002). 


\section{References}

Annegarn J, Spruit M A, Uszko-Lencer N H, Vanbelle S, Savelberg H H, Schols A M, Wouters E F and Meijer K 2011 Objective physical activity assessment in patients with chronic organ failure: a validation study of a new single-unit activity monitor Arch. Phys. Med. Rehabil. 921852

Berendsen B A J, Hendiks M R C, Meijer K, Plasqui G, Schaper N C and Savelberg H H C M 2014 Which activity monitor to use? Validity, reproducibility and user friendliness of three activity monitor. BMC Public Health 14749

Berendsen B A, Hendriks M R, Verhagen E A, Schaper N C, Kremers S P and Savelberg H H 2011 Effectiveness and cost-effectiveness of 'BeweegKuur', a combined lifestyle intervention in the Netherlands: rationale, design and methods of a randomized controlled trial BMC Public Health 11815

Choi L, Liu Z, Matthews C E and Buchowski M S 2011 Validation of accelerometer wear and nonwear time classification algorithm Med. Sci. Sports Exerc. 43 357-64

Choi L, Ward S C, Schnelle J F and Buchowski M S 2012 Assessment of wear/nonwear time classification algorithms for triaxial accelerometer Med. Sci. Sports Exerc. 44 2009-16

Evenson K R and Terry J W Jr 2009 Assessment of differing definitions of accelerometer nonwear time Res. Quart. Exerc. Sport 80 355-62

Harrington D M, Dowd K P, Bourke A K and Donnelly A E 2011 Cross-sectional analysis of levels and patterns of objectively measured sedentary time in adolescent females Int. J. Behav. Nutr. Phys. Act. 8120

Healy G N, Dunstan D W, Salmon J, Cerin E, Shaw J E, Zimmet P Z and Owen N 2008 Breaks in sedentary time: beneficial associations with metabolic risk Diabetes Care 31 661-6

Hutto B, Howard V J, Blair S N, Colabianchi N, Vena J E, Rhodes D and Hooker S P 2013 Identifying accelerometer nonwear and wear time in older adults Int. J. Behav. Nutr. Phys. Act. 10120

Masse L C, Fuemmeler B F, Anderson C B, Matthews C E, Trost S G, Catellier D J and Treuth M 2005 Accelerometer data reduction: a comparison of four reduction algorithms on select outcome variables Med. Sci. Sports Exerc. 37 S544-54

Miller G D, Jakicic J M, Rejeski W J, Whit-Glover M C, Lang W, Walkup M P and Hodges M L 2013 Effect of varying accelerometry criteria on physical activity: the look ahead study Obesity (Silver Spring) 21 32-44

Pat Rapp M, Nelson F, Oliver M, Bergstrom N and Cron S G 2010 Comparison of commonly used placement sites for activity monitoring Biol. Res. Nurs. 11 302-9

Rosenberger M E, Haskell W L, Albinali F, Mota S, Nawyn J and Intille S 2013 Estimating activity and sedentary behavior from an accelerometer on the hip or wrist Med. Sci. Sports Exerc. 45 964-75

Troiano R P, Berrigan D, Dodd K W, Masse L C, Tilert T and McDowell M 2008 Physical activity in the United States measured by accelerometer Med. Sci. Sports Exerc. 40 181-8

Trost S G, McIver K L and Pate R R 2005 Conducting accelerometer-based activity assessments in fieldbased research Med. Sci. Sports Exerc. 37 S531-43

Tudor-Locke C, Brashear M M, Johnson W D and Katzmarzyk P T 2010 Accelerometer profiles of physical activity and inactivity in normal weight, overweight, and obese U.S. men and women Int. J. Behav. Nutr. Phys. Act. 760

Van Domelen D R, Koster A and Harris T B 2011 Accelerometer nonwear algorithms: optimizing parameters for both wear states Med. Sci. Sports Exerc. 43932 author reply 933

Winkler E A, Gardiner P A, Clark B K, Matthews C E, Owen N and Healy G N 2012 Identifying sedentary time using automated estimates of accelerometer wear time Br. J. Sports Med. 46 436-42

Zhang S, Rowlands A V, Murray P and Hurst T L 2012 Physical activity classification using the GENEA wrist-worn accelerometer Med. Sci. Sports Exerc. 44 742-8 